\title{
Ciclo de mejora en el aula para la elaboración de un plan de mantenimiento bajo el método RCM
}

\section{Improvement cycle in classroom for the development of a maintenance plan using RCM method}

ANTONIO SÁNCHEZ HERGUEDAS

ORCID: https://orcid.org/0000-0001-5135-3250

Universidad de Sevilla. Departamento de

Organización Industrial y Gestión de Empresas I antoniosh@us.es

Fecha de recepción: 28-05-2019

Fecha de aceptación: 10-06-2019

DOI: http://dx.doi.org/10.12795/9788447221912.021

Pp.: 494-520 


\section{Resumen}

Este Ciclo de Mejora en el Aula (CIMA) aplica una innovación al proceso de aprendizaje de los alumnos de Ingeniería, al utilizar el método RCM para la elaboración de un plan de mantenimiento. Desde la exposición teórica tradicional se pretende innovar utilizando actividades relacionadas con aspectos básicos de la formación perseguida, intentando que el alumno construya su conocimiento relacionando casos de uso cotidiano y contenidos de la disciplina. Se ha elaborado un mapa de contenidos, a partir del cual se diseña cada actividad para provocar el dinamismo del alumno, compaginando el debate con la investigación. Para evaluar el nivel de conocimiento de los alumnos se desarrolla un cuestionario, estableciendo en cada pregunta varios niveles de aprendizaje, escalonados por algún obstáculo de conocimiento. Los resultados se expresan gráficamente, estableciendo las diferencias entre el punto de partida y el final del ciclo.

Palabras Clave: Sistemas de gestión del mantenimiento; Grado en Ingeniería de Organización Industrial; Docencia universitaria; Experimentación docente universitaria; RCM (Mantenimiento Centrado en la Fiabilidad).

\section{Abstract}

This Improvement Cycle in Classroom (ICIC) constitutes an innovation to the learning process of Engineering students, by using the RCM method to develop a maintenance plan. Progressing from the traditional theoretical teaching method, we adopt an activity based teaching process which encourages the students to participate in classroom. This enables them to develop their own approach to the subject relating day-to-day cases to the subject contents. A map of contents has been prepared in order to design activities to encourage the use of the student's own initiative, combining the discussion with the investigation. A questionnaire has been developed to assess the level of knowledge of the students. Several levels of learning have been established for each question, challenging students to progress through these. The results are expressed graphically, clearly setting out the progress achieved between the commencement and conclusion of the cycle.

Keywords: Maintenance management systems; Degree in Industrial Organization Engineering; University teaching; University teaching experimentation; RCM (Reliability Centered Maintenance). 


\section{Descripción del contexto}

La asignatura Sistemas de Gestión del Mantenimiento está ubicada en el tercer curso del Grado de Ingeniería de Organización Industrial (GIOI). En el curso 2018/19 se han matriculado 46 alumnos, 6 de ellos repetidores. De ellos, sólo 42 han participado en este Ciclo de Mejora en el Aula (CIMA), pero únicamente 35 de ellos lo han completado en su totalidad (76\%): asistencia, entrega de trabajos y cumplimentación de cuestionarios. Este CICA se desarrolla durante ocho horas en dos semanas de calendario, distribuidas en seis sesiones de ochenta minutos.

El objetivo de la asignatura es que los alumnos sean capaces de implementar los distintos sistemas de gestión de mantenimiento, así como las técnicas que se utilizan en su diseño y desarrollo. Este ciclo se ha centrado en el aprendizaje del diseño y mejora de un plan de mantenimiento de una instalación industrial, aplicando el método RCM (Reliability Centered Maintenance / Mantenimiento Centrado en la Fiabilidad) a los equipos que componen esa instalación.

\section{Diseño del Ciclo de Mejora en el Aula}

Para determinar qué mantenimiento es el requerido en su contexto operativo por cada uno de los equipos que componen una instalación, los alumnos deben aprender a aplicar el método RCM. El alumno parte de unos conocimiento previos adquiridos en sesiones anteriores, que le ayudarán a comprender y valorar los contenidos que se van a trabajar en este CIMA. 


\section{Mapa de contenidos}

En nuestra actuación intentamos aunar conceptos, procedimientos, actitudes y valores (Bain, 2007); para ello se ha desarrollado un mapa de contenidos que pretende representar el proceso de selección del tipo de mantenimiento, siguiendo el método RCM, para cada modo de fallo. En el mapa de la Figura 1 se representa este proceso que se inicia con la información procedente del GMAO (Gestión del Mantenimiento Asistido por Ordenador), determinando los distintos tipos de fallos funcionales que afectan a la instalación o equipos, y, a partir de ellos, los posibles modos de fallos.

Cada modo de fallo será analizado utilizando la técnica AMFE (Análisis de los Modos de Fallos y sus Efectos), apoyándose en otras técnicas como los árboles de fallo, árboles de decisión, análisis de fiabilidad y análisis de Weibull. Como resultado se establecerá el listado de modos de fallo, relacionado con sus consecuencias (efectos) y la causa origen de cada uno.

Posteriormente, se valorará el riesgo de aparición del fallo para cada modo de fallo. Este riesgo se mide en grados de criticidad, que depende de la probabilidad de ocurrir el fallo y de la severidad de sus consecuencias (técnica AMFEC, Análisis de los Modos de Fallos, sus Efectos y su Criticidad). El grado de criticidad adjudicado a cada modo de fallo posicionará a cada uno en un ranking de prioridad de actuación.

Comenzando por el modo de fallo de mayor criticidad, se seleccionará la tarea de mantenimiento más eficiente. Primero se debe tener en cuenta la posibilidad de aplicar algunas técnicas de análisis de la condición. Si existe una señal que anuncie el fallo, se implantará la técnica que analice esa condición (monitorización de la señal), posponiendo la actuación de mantenimiento justo antes de 
que aparezca el fallo. A este tipo de actuaciones les llamamos de mantenimiento predictivo ( $P d M)$. Si no existe esa señal se establecerán tareas de mantenimiento por edad, las llamadas tareas de mantenimiento preventivo (PM). Por último, para aquellos modos de fallo de baja criticidad, no se planificará ninguna tarea y se esperará a que ocurra el fallo (RTF).

Una vez establecida la nueva política de mantenimiento (suma de las tareas previstas para cada modo de fallo), se incorporarán a las actividades de mantenimiento, completando así un el ciclo de mejora del plan de mantenimiento de la instalación. El responsable de diseñar estas políticas es el ingeniero de mantenimiento, al que se pretende formar en esta asignatura.

En la Figura 1, aparecen en verde los contenidos procedimentales que reflejan la metodología RCM para la selección del tipo de tarea de mantenimiento. En rojo los contenidos actitudinales que involucran al alumno a la utilización de estas técnicas, mientras que los conceptuales, señalados en azul, han sido considerados como insertados, de forma implícita, junto a algunos contenidos procedimentales y actitudinales, entre los que destacan el modo de fallo, la criticidad, análisis de la condición y la información.

Jornadas de Formación e Innovación Docente del Profesorado | № 2 (2019) Esta obra se distribuye con la licencia Creative Commons Reconocimiento-NoComercial-SinObraDerivada Internacional (CC BY-NC-ND 4.0.) 


\section{ANTONIO SÁNCHEZ HERgUEdAS}

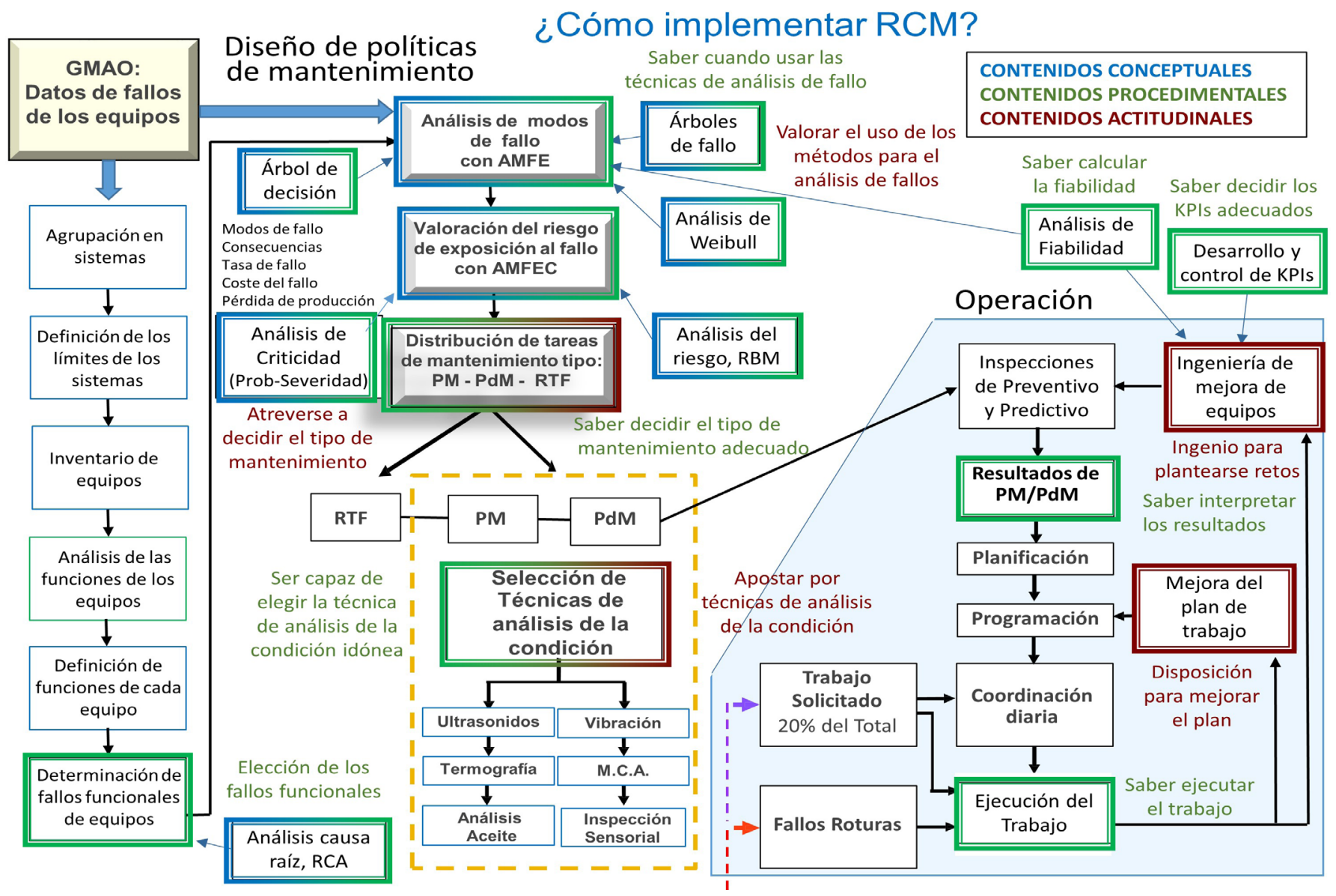

Figura 1. Mapa de contenidos: Diseño de un plan de mantenimiento.

Jornadas de Formación e Innovación Docente del Profesorado I № 2 (2019) Esta obra se distribuye con la licencia Creative Commons Reconocimiento-NoComercial-SinObraDerivada Internacional (CC BY-NC-ND 4.0.) 


\section{Modelo metodológico}

Tradicionalmente el método RCM se ha impartido apoyándose en la exposición teórica de los contenidos, incluyéndose la práctica mediante la resolución de problemas. Ahora se pretende evolucionar a un modelo metodológico basado en la reelaboración de ideas de los alumnos, ampliando este aprendizaje por investigación durante la segunda parte de las actividades (De Alba y Porlán, 2017; Delval, 2001).

Se asume que la construcción del conocimiento es susceptible de ser reelaborada y reformada y que el alumno aprende mediante la reorganización de sus esquemas preexistentes a partir de la interacción con informaciones externas. Se pretende, pues, que la información llegue al alumno en forma de actividades, debate y documentos de trabajo. Por eso, cada actividad incluye los contenidos de un apartado del proceso RCM. Además en su diseño se han incluido los nexos con las actividades precedentes y posteriores, para no sólo servir de vehículo de construcción del conocimiento de ese apartado, sino ayudar a construir la red de conexiones del proceso completo del método RCM. La secuencia de actividades propuesta es la que seguiría una empresa que realiza su estudio de mejora de planes de mantenimiento.

El proceso cíclico de aprendizaje a seguir sería el siguiente:

1. Planteamiento de un problema o preguntas por parte del profesor.

2. Toma de conciencia y expresión de ideas por parte de los alumnos.

3. Puesta en práctica de actividades de contraste que pongan en cuestión la ideas iniciales.

4. Elaboración de conclusiones sobre el proceso de transición desde ideas iniciales a finales. 
Para llevar a cabo este modelo, el profesor establece la serie de actividades (Finkel, 2008) ordenadas de acuerdo al método RCM, basadas en preguntas y problemas, como la identificación de los modos de fallos, las causas y su criticidad, las decisiones, la condición de los equipos y la necesidad de información. Mediante las actividades se abrirá debate entre los alumnos, la misión el profesor será guiarlo (Bain, 2007), para asegurar que las ideas resultantes de la discusión correspondan con los objetivos de enseñanza prevista.

Previamente a la primera actividad se pedirá a los alumnos que rellenen un cuestionario que servirá para establecer su nivel de conocimiento antes del inicio del ciclo de mejora y contrastarlo al final del ciclo con las ideas evolucionadas. De esta manera se podrá establecer una evaluación del proceso de aprendizaje de cada alumno y del grupo en general.

\section{Secuencia de actividades programadas}

En la secuencia de actividades, al principio se trabaja sobre las necesidades de información, luego sobre la metodología del RCM y las técnicas que utiliza, para posteriormente establecer prioridades, controles y acabar con la medición y el origen de la información.

Se proponen diez actividades, expuestas en la Tabla 1 , con una duración de 40 minutos cada una. 
Tabla 1. Secuencia de actividades y objetivo deseado.

\begin{tabular}{|c|c|c|}
\hline № & Actividades & Objetivo de las actividades \\
\hline 1 & $\begin{array}{l}\text { Análisis DAFO de la com- } \\
\text { pañía para la cual vamos } \\
\text { a aplicar un estudio RCM. }\end{array}$ & $\begin{array}{l}\text { Que los alumnos comprendan el } \\
\text { contexto empresarial donde se van a } \\
\text { plantear los problemas. Deben cono- } \\
\text { cer el equipo, analizando su compo- } \\
\text { sición, funciones y uso. }\end{array}$ \\
\hline 2 & $\begin{array}{l}\text { Repaso general del mé- } \\
\text { todo RCM aplicado a } \\
\text { Aerogeneradores. }\end{array}$ & $\begin{array}{l}\text { Que los alumnos aprendan cómo se } \\
\text { diseñan las actividades de manteni- } \\
\text { miento, desde la óptica de la fiabili- } \\
\text { dad, considerando modos, causas y } \\
\text { consecuencias de los fallos. }\end{array}$ \\
\hline 3 & Árboles de decisión. & $\begin{array}{l}\text { Que aprendan a construir un árbol } \\
\text { de decisiones. }\end{array}$ \\
\hline 4 & $\begin{array}{l}\text { Adaptación de un histo- } \\
\text { grama a una función de } \\
\text { densidad teórica. }\end{array}$ & $\begin{array}{l}\text { Que los alumnos conozcan el pro- } \\
\text { ceso de elaboración de informa- } \\
\text { ción a partir de datos de fallo. Se } \\
\text { pretende encontrar una función de } \\
\text { distribución que represente el com- } \\
\text { portamiento de un equipo ante el } \\
\text { fallo. }\end{array}$ \\
\hline 5 & Análisis de vibraciones. & $\begin{array}{l}\text { Que valoren la información técnica } \\
\text { que ofrece el análisis de vibraciones, } \\
\text { adopten el concepto de condición } \\
\text { del equipo y lo relacionen con el } \\
\text { tiempo previo a la aparición del fallo. }\end{array}$ \\
\hline 6 & $\begin{array}{l}\text { Tipos de mantenimiento y } \\
\text { otras técnicas de mante- } \\
\text { nimiento predictivo. }\end{array}$ & $\begin{array}{l}\text { Que los alumnos identifiquen el } \\
\text { mantenimiento que requiere cada } \\
\text { equipo en función a la condición que } \\
\text { se pueda monitorizar. }\end{array}$ \\
\hline 7 & Mantenimiento y riesgo. & $\begin{array}{l}\text { Que comprendan el concepto de } \\
\text { riesgo, que lo asocie a la criticidad } \\
\text { de un modo de fallo y establezcan } \\
\text { un ranking de estos en función la } \\
\text { criticidad. }\end{array}$ \\
\hline 8 & $\begin{array}{l}\text { Análisis de la causa raíz, } \\
\text { RCA. }\end{array}$ & $\begin{array}{l}\text { Que los alumnos se conciencien } \\
\text { acerca de que siempre que existe un } \\
\text { fallo hay una causa que lo origina. Es } \\
\text { fundamental descubrir la causa que } \\
\text { lo origina y eliminarla, para evitar } \\
\text { que se vuelva a repetir. }\end{array}$ \\
\hline
\end{tabular}




\begin{tabular}{|c|l|l|}
\hline 9 & $\begin{array}{l}\text { KPIs y la información en } \\
\text { Mantenimiento. }\end{array}$ & $\begin{array}{l}\text { Que los alumnos comprendan que } \\
\text { es más fácil mejorar cuando algo se } \\
\text { mide. Deben buscar indices para dis- } \\
\text { tintas actividades a partir de da- } \\
\text { tos e información, compararlos y ver } \\
\text { tendencias. }\end{array}$ \\
\hline 10 & $\begin{array}{l}\text { Gestión del Manteni- } \\
\text { miento Asistido por Orde- } \\
\text { nador, GMAO. }\end{array}$ & $\begin{array}{l}\text { Que aprendan las distintas tareas } \\
\text { que es capaz de realizar un GMAO y } \\
\text { para qué se utiliza esa información. }\end{array}$ \\
\hline
\end{tabular}

Durante el desarrollo de las actividades, en la fase de discusiones de cada actividad, se plantea el problema de estudio, realizado y ejecutado para un importante fabricante de aerogeneradores.

En cada actividad se ha intentado abarcar uno de los aspectos básicos del proceso de elaboración de un plan de mantenimiento, como la determinación de los modos de fallo, el cálculo de su criticidad, las técnicas de análisis de la condición aplicables y cómo medir la evolución del mantenimiento y el diseño de la recogida de información. Para ello, en las actividades se presentan ejemplos generales y cotidianos relacionándolos con ejemplos particulares y reales de la disciplina.

\section{Evaluación del Ciclo de Mejora en el Aula}

Con objeto aproximarme a la evaluación del aprendizaje, se ha desarrollado un cuestionario de preguntas (Rivero y Porlán, 2017)la formación docente debe ser un objetivo de máxima prioridad en la Educación Superior. Debe ir más allá de actividades puntuales y ha de basarse en programas de largo alcance que abarquen todas las dimensiones didácticas (fines, contenidos, metodología y evaluación, pasado a los alumnos en el momento inicial y final de este CIMA. Este cuestionario pretende explorar cuestiones centrales del contenido a enseñar. Se 
intenta descubrir: déficit de conceptos, obstáculos en la comprensión, niveles de conocimientos, formas de razonar, ideas, actitudes, perjuicios e intereses. Otro objetivo perseguido es proporcionar orientación para ir adecuando la propuesta de contenidos y las actividades que se desarrollarán posteriormente.

El cuestionario, expresado en la Tabla 2, se utilizará también para valorar el aprendizaje. Al iniciar el ciclo se pedirá a los alumnos que contesten a las ocho preguntas del cuestionario (inicial). Una vez finalizado el ciclo, tras la actividad 10 , se volverá a pedir a los alumnos que cumplimenten el mismo cuestionario (final).

Tabla 2. Cuestionario inicial y final.

\begin{tabular}{|c|l|}
\hline 1 & $\begin{array}{l}\text { ¿Qué información relevante para optimizar el mantenimiento pode- } \\
\text { mos sacar de un Sistema de Gestión Asistido por Ordenador, GMAO? }\end{array}$ \\
\hline 2 & ¿Qué son los fallos funcionales y cómo afectan al mantenimiento? \\
\hline 3 & $\begin{array}{l}\text { ¿De qué técnicas disponemos y qué nos aportan al analizar cada } \\
\text { modo de fallo? }\end{array}$ \\
\hline 4 & $\begin{array}{l}\text { ¿De qué se compone la criticidad y cómo afecta su grado al trata- } \\
\text { miento de un modo de fallo? }\end{array}$ \\
\hline 5 & $\begin{array}{l}\text { ¿Qué entiendes por condición de un equipo? ¿Cómo la medirías? } \\
\text { ¿Dónde coloco las alarmas? }\end{array}$ \\
\hline 6 & $\begin{array}{l}\text { ¿Qué relación existe entre técnicas de análisis de la condición y } \\
\text { técnicas de análisis de fallos? }\end{array}$ \\
\hline 7 & $\begin{array}{l}\text { Define "técnicas de análisis de la condición". ¿En qué casos están } \\
\text { indicadas? }\end{array}$ \\
\hline 8 & $\begin{array}{l}\text { Distintos tipos de tareas de mantenimiento. ¿Cómo se decide cuál } \\
\text { es la más conveniente para cada equipo? }\end{array}$ \\
\hline
\end{tabular}

Jornadas de Formación e Innovación Docente del Profesorado | № 2 (2019) Esta obra se distribuye con la licencia Creative Commons Reconocimiento-NoComercial-SinObraDerivada Internacional (CC BY-NC-ND 4.0.) 


\section{Aplicación del Ciclo de Mejora en el Aula}

Durante la aplicación del CIMA, la clase se ha dividido en seis grupos de siete alumnos que van a participar en las actividades. El motivo de la división es el establecimiento de debate durante la segunda parte de cada actividad. Inicialmente se expondrán las actividades, se debatirán en grupos, se expondrán los aspectos más relevantes tratados en cada grupo y se debatirá finalmente en el conjunto de la clase.

\section{Desarrollo de las actividades}

A continuación se muestra en la Tabla 3 el resumen del desarrollo de las actividades del CIMA.

Tabla 3. Desarrollo de las actividades durante el ciclo de mejora.

\begin{tabular}{|c|l|l|}
\hline No & \multicolumn{1}{|c|}{ Actividades } & \multicolumn{1}{c|}{ Desarrollo de actividades } \\
\hline 1 & $\begin{array}{l}\text { Análisis DAFO de } \\
\text { la compañía para } \\
\text { la cual vamos a } \\
\text { aplicar un estu- } \\
\text { dio RCM. }\end{array}$ & $\begin{array}{l}\text { Se contextualiza una empresa fabricante de ae- } \\
\text { rogeneradores y la composición y descripción } \\
\text { funcional de un aerogenerador. Se relata una } \\
\text { aventura, se plantea un problema y se expone } \\
\text { una relación de preguntas para focalizar la aten- } \\
\text { ción en el generador. Luego durante la segunda } \\
\text { parte de la actividad, se entrega a los alumnos un } \\
\text { análisis DAFO de la empresa, un documento so- } \\
\text { bre la composición de un aerogenerador y una } \\
\text { encuesta de efectividad del mantenimiento (MES). } \\
\text { Tras analizarlos comienza el debate en cada } \\
\text { grupo, posteriormente se exponen los resultados } \\
\text { a la clase. }\end{array}$ \\
\hline
\end{tabular}

Jornadas de Formación e Innovación Docente del Profesorado | № 2 (2019) Esta obra se distribuye con la licencia Creative Commons Reconocimiento-NoComercial-SinObraDerivada 4.0 Internacional (CC BY-NC-ND 4.0.) 


\begin{tabular}{|l|l|l|}
\hline Repaso gene- & $\begin{array}{l}\text { Se trabajan contenidos de fiabilidad: modos, cau- } \\
\text { ral del método } \\
\text { RCM aplicado a consecuencias de los fallos. Se utilizan } \\
\text { aerogeneradores. } \\
\text { ejemplos que permiten razonar los aspectos teó- } \\
\text { ricos. Se hace hincapié en que para algunos mo- } \\
\text { dos de fallo, la probabilidad de fallar no aumenta } \\
\text { con la edad, pero en el caso de que así sea, se } \\
\text { tratará de evitar que los equipos envejezcan (sus- } \\
\text { tituyendo convenientemente las piezas de des- } \\
\text { gaste). Se discute en grupo y se exponen las } \\
\text { conclusiones. }\end{array}$ \\
\hline $\begin{array}{l}\text { Árboles de } \\
\text { decisión. }\end{array}$ & $\begin{array}{l}\text { Se plantea la construcción de un árbol de de- } \\
\text { cisión, para distintos tipos de reforma de un } \\
\text { edificio. Se revelan las distintas opciones, carac- } \\
\text { terizadas por la probabilidad de ocurrir y la ga- } \\
\text { nancia que ofrece cada una. En base a estos } \\
\text { conceptos se evalúa la ventaja de una opción } \\
\text { frente a las demás. Posteriormente se trabaja so- } \\
\text { bre el árbol de decisión del generador de un ae- } \\
\text { rogenerador. El alumno establecer las relaciones } \\
\text { entre componentes, subcomponentes, función, } \\
\text { fallo funcional, modo de fallo, consecuencias, } \\
\text { tasa de fallo, coste y pérdida de producción. Se } \\
\text { discute en grupo y se exponen las conclusiones. }\end{array}$ \\
\hline $\begin{array}{l}\text { Adaptación de un } \\
\text { histograma a una } \\
\text { función de densi- } \\
\text { dad teórica. }\end{array}$ & $\begin{array}{l}\text { Se presenta un ejercicio donde se propone a los } \\
\text { alumnos la construcción de un histograma y la } \\
\text { curva que mejor se adapte a su forma geomé- } \\
\text { trica. Se pide comparar la curva obtenida con } \\
\text { una función matemática (función de densidad) } \\
\text { y determinar los parámetros de ésta. Posterior- } \\
\text { mente se entrega un documento sobre la proba- } \\
\text { bilidad de fallo del generador y su relación con } \\
\text { el comportamiento de los equipos (fiabilidad) y } \\
\text { un segundo documento de aplicación práctica de } \\
\text { ajuste de datos de fallo a una función de distri- } \\
\text { bución de Weibull. Se discute en grupo y se expo- } \\
\text { nen las conclusiones. }\end{array}$ \\
\hline & &
\end{tabular}

Jornadas de Formación e Innovación Docente del Profesorado | № 2 (2019) Esta obra se distribuye con la licencia Creative Commons 


\begin{tabular}{|c|c|c|}
\hline 5 & $\begin{array}{l}\text { Análisis de } \\
\text { vibraciones. }\end{array}$ & $\begin{array}{l}\text { Se plantea el problema de cómo un observador, } \\
\text { que está al borde de una piscina, intenta detec- } \\
\text { tar cuándo va a llegar la ola. El problema se hace } \\
\text { más complejo cuando se incorporan nuevas olas } \\
\text { con distintos orígenes. Los alumnos conciben el } \\
\text { problema de determinar la vibración haciendo la } \\
\text { analogía con las olas. Luego se entrega un docu- } \\
\text { mento donde se recogen conceptos relacionados } \\
\text { con vibraciones, espectros y patrones, alarmas } \\
\text { y la captura e interpretación de las medidas. Se } \\
\text { discute y exponen conclusiones. }\end{array}$ \\
\hline 6 & $\begin{array}{l}\text { Tipos de man- } \\
\text { tenimiento y } \\
\text { otras técnicas de } \\
\text { mantenimiento } \\
\text { predictivo. }\end{array}$ & $\begin{array}{l}\text { Se plantean distintas preguntas que revelan los } \\
\text { distintos tipos de mantenimiento. Se relacionan } \\
\text { con el conocimiento de la condición de los mo- } \\
\text { dos de fallo, el desgaste por edad y la necesidad } \\
\text { de rediseñar. Se analiza y discute un documento } \\
\text { donde se analizan estos tipos de mantenimiento } \\
\text { y las técnicas predictivas: termografia, análisis de } \\
\text { aceite, inspecciones, test eléctrico y vibraciones. } \\
\text { Se discute y exponen conclusiones. }\end{array}$ \\
\hline 7 & $\begin{array}{l}\text { Mantenimiento y } \\
\text { riesgo. }\end{array}$ & $\begin{array}{l}\text { Se plantea el riesgo del posible impacto de un } \\
\text { meteorito sobre la Tierra y que el cárter de un } \\
\text { motor quede sin aceite. Se considera la proba- } \\
\text { bilidad de que estos eventos ocurran y la severi- } \\
\text { dad con que afectarían a la superficie terrestre o } \\
\text { al motor. Se introduce la matriz de criticidad para } \\
\text { clasificar el riesgo de un modo de fallo, como } \\
\text { producto de la probabilidad y severidad. Se ana- } \\
\text { liza un documento sobre el mantenimiento ba- } \\
\text { sado en el riesgo, aplicado a aerogeneradores. Se } \\
\text { discute y exponen conclusiones. }\end{array}$ \\
\hline 8 & $\begin{array}{l}\text { Análisis de la } \\
\text { causa raíz, RCA. }\end{array}$ & $\begin{array}{l}\text { Se plantea a los alumnos descubrir la causa raíz } \\
\text { de la destrucción del transbordador Columbia, } \\
\text { durante la reentrada en la atmósfera. Se mues- } \\
\text { tra cómo se realiza el análisis, cuál fue la causa } \\
\text { y cómo se llegó a ella. Luego se analiza la téc- } \\
\text { nica seguida, RCA (análisis causa raíz) y se aplica } \\
\text { al caso de los modos de fallo presentados en el } \\
\text { generador, distinguiendo entre causa origen y } \\
\text { otras causas secundarias. Se discute y exponen } \\
\text { conclusiones. }\end{array}$ \\
\hline
\end{tabular}

Jornadas de Formación e Innovación Docente del Profesorado | № 2 (2019) Esta obra se distribuye con la licencia Creative Commons Reconocimiento-NoComercial-SinObraDerivada Internacional (CC BY-NC-ND 4.0.) 


\begin{tabular}{|l|l|l|}
\hline 9 & $\begin{array}{l}\text { KPIs y la in- } \\
\text { formación en } \\
\text { Mantenimiento. }\end{array}$ & $\begin{array}{l}\text { Se expone la necesidad de saber cómo se está } \\
\text { actuando en mantenimiento. El alumno debe } \\
\text { comprender que se necesita conocer el estado } \\
\text { actual y la tendencia, a partir de los datos de fa- } \\
\text { llo recogidos en el GMAO. Comprender y saber } \\
\text { desarrollar los KPIs es fundamental para con- } \\
\text { trolar el mantenimiento realizado. Se analiza un } \\
\text { documento con KPIs para el control del manteni- } \\
\text { miento de los aerogeneradores. Se discute y ex- } \\
\text { ponen conclusiones. }\end{array}$ \\
\hline $\begin{array}{l}\text { GMAO, Gestión } \\
\text { del Manteni- } \\
\text { miento Asistido } \\
\text { por Ordenador. }\end{array}$ & $\begin{array}{l}\text { Se plantean los distintos bloques que compo- } \\
\text { nen un GMAO, cómo se adquieren los datos y cuál } \\
\text { es la utilidad de la información elaborada. Se re- } \\
\text { laciona esta información con las diferentes téc- } \\
\text { nicas de análisis de los fallos. Posteriormente se } \\
\text { accede a la página web de un desarrollador de } \\
\text { GMAOs, donde se detalla cada una de las tareas } \\
\text { que puede realizar esta herramienta. Se discute y } \\
\text { exponen conclusiones. }\end{array}$ \\
\hline
\end{tabular}

\section{Avances conseguidos}

A mi juicio personal, los avances conseguidos en este CIMA han sido importantes: la actitud frente al desafio, la forma de trabajar y los contenidos adquiridos, observados durante las sesiones y en momentos posteriormente al CIMA así me lo atestiguaron. Algunos de estos avances fueron:

- La clase está perfectamente integrada en el modelo metodológico seguido, ampliando el aprendizaje por investigación (De Alba y Porlán, 2017)la formación docente debe ser un objetivo de máxima prioridad en la Educación Superior. Debe ir más allá de actividades puntuales y ha de basarse en programas de largo alcance que abarquen todas las dimensiones didácticas (fines, contenidos, metodología y evaluación al final de cada actividad.

- Los alumnos son colaboradores y proponen acciones, apareciendo temas de debate de nivel superior al que se había predefinido. La clase transmite espíritu optimista. 
- La teoría está mejor aprendida, ya que las preguntas de los alumnos durante los debates y la exposición así lo corroboran, e igualmente la superación de algunos obstáculos por parte de la mayoría.

- Las clases, aunque han requerido un considerable esfuerzo de preparación por parte del docente, han sido más fáciles de coordinar; la incertidumbre inicial ante el cambio se convirtió en un final relajado.

- Durante las actividades se estableció una sana competencia entre grupos, intentando exponer las mejores aportaciones.

- La actividad propuesta a entregar en el mismo día, reseña de la sesión, ha sido muy instructiva. Ha obligado a los alumnos a escribir sus razonamientos de la actividad, lo cual implica una fijación importante de ideas y mejorar la posibilidad de actualizarlas en el futuro (Esteve Zarazaga, 2007)J. y Esteve, J. M., 2001. La mayoría contestó en el día, lo cual ayudó a conseguir esta meta.

\section{Dificultades encontradas y otros aspectos}

No siempre tuve el $100 \%$ de asistencia del alumnado, faltando dos o tres alumnos por sesión (menos del 10\%). En todo caso, el contenido de las actividades se puso a disposición de los no asistentes, de modo que pudieran completarlas de forma individual, intentando que estos alumnos siguieran conectados. A todos se les exigió la reseña de la actividad.

Las actividades han sido motivadoras, pero es cierto que algunas más que otras, por ejemplo la actividad no 6 , caída del meteorito, produjo gran interés en la clase. Otras como la no 3, árboles de decisión, fue difícil de entender. 


\section{Evaluación del aprendizaje de los estudiantes}

Para conocer la evolución del aprendizaje de los alumnos se recurre a la evaluación de los cuestionarios inicial y final. Se establecen distintos niveles de conocimiento para cada cuestión del cuestionario, separados por algún obstáculo, hipotético, de aprendizaje. El superar ese obstáculo y alcanzar un nivel superior supondrá una mejor valoración para el alumno. Se utilizan para ello escaleras de aprendizaje (Rivero y Porlán, 2017)la formación docente debe ser un objetivo de máxima prioridad en la Educación Superior. Debe ir más allá de actividades puntuales y ha de basarse en programas de largo alcance que abarquen todas las dimensiones didácticas (fines, contenidos, metodología y evaluaciónla formación docente debe ser un objetivo de máxima prioridad en la Educación Superior. Debe ir más allá de actividades puntuales y ha de basarse en programas de largo alcance que abarquen todas las dimensiones didácticas (fines, contenidos, metodología y evaluación como herramienta de evaluación, con cinco niveles por cuestión (Tabla 4).

Jornadas de Formación e Innovación Docente del Profesorado | № 2 (2019) Esta obra se distribuye con la licencia Creative Commons 
Tabla 4. Niveles en las escaleras de aprendizaje y obstáculos a superar.

\begin{tabular}{|c|c|c|}
\hline C & Escalera de aprendizaje & $\begin{array}{l}\text { Obstáculos para ascender al } \\
\text { escalón siguiente }\end{array}$ \\
\hline 1 & $\begin{array}{l}\text { 1. No responde nada o la con- } \\
\text { testación no concuerda. } \\
\text { 2. Se puede sacar información } \\
\text { de los datos de fallos. } \\
\text { 3. Se puede obtener un histó- } \\
\text { rico de los modos de fallos. } \\
\text { 4. Información de los modos } \\
\text { de fallo para planificar el } \\
\text { mantenimiento. } \\
\text { Información para la ges- } \\
\text { tión del mantenimiento a } \\
\text { nivel operativo, táctico y } \\
\text { estratégico. }\end{array}$ & $\begin{array}{l}1 \rightarrow 2 \text { GMAO como suministrador } \\
\text { de información. } \\
2 \rightarrow 3 \text { Información clasificada de } \\
\text { acuerdo a los modos de fallo. } \\
3 \rightarrow 4 \text { Información utilizada para } \\
\text { planificar el mantenimiento. } \\
4 \rightarrow 5 \text { Información utilizada } \\
\text { a nivel operativo, táctico y } \\
\text { estratégico. }\end{array}$ \\
\hline 2 & $\begin{array}{l}\text { 1. No responde nada o la con- } \\
\text { testación no concuerda. } \\
\text { 2. Definición de fallo. } \\
\text { 3. Definición de fallo funcional. } \\
\text { 4. La ingeniería de manteni- } \\
\text { miento busca el origen del } \\
\text { fallo. } \\
\text { 5. En ingeniería de manteni- } \\
\text { miento se estudia el modo } \\
\text { de fallo, determinando el } \\
\text { tipo de mantenimiento re- } \\
\text { querido por el equipo. }\end{array}$ & $\begin{array}{l}1 \rightarrow 2 \text { El concepto de fallo. } \\
2 \rightarrow 3 \text { Concepto de fallo funcional. } \\
3 \rightarrow 4 \text { Buscar el origen del fa- } \\
\text { llo, base para la resolución de } \\
\text { problemas. } \\
4 \rightarrow 5 \text { Análisis de los modos de fa- } \\
\text { llo para determinar el tipo de } \\
\text { mantenimiento requerido por el } \\
\text { equipo. }\end{array}$ \\
\hline 3 & $\begin{array}{l}\text { 1. No responde nada o la con- } \\
\text { testación no concuerda. } \\
\text { 2. Cita algunas técnicas para } \\
\text { analizar modos de fallo. } \\
\text { 3. Cita y define algunas } \\
\text { técnicas. } \\
\text { 4. Cita y define la mayoría de } \\
\text { las técnicas. } \\
\text { Cita y define todas las } \\
\text { técnicas. }\end{array}$ & $\begin{array}{l}1 \rightarrow 2 \text { Conoce algunas de las } \\
\text { técnicas. } \\
2 \rightarrow 3 \text { Conoce y sabe definir } \\
\text { algunas. } \\
3 \rightarrow 4 \text { Conoce y sabe definir } \\
\text { muchas. } \\
4 \rightarrow 5 \text { Conoce, sabe definir y apli- } \\
\text { car todas las técnicas. }\end{array}$ \\
\hline
\end{tabular}




\begin{tabular}{|c|c|c|}
\hline 4 & $\begin{array}{l}\text { 1. No responde nada o la con- } \\
\text { testación no concuerda. } \\
\text { 2. Define la criticidad. } \\
\text { 3. Define los componentes de } \\
\text { la criticidad. } \\
\text { 4. Relaciona la criticidad de un } \\
\text { modo de fallo con el riesgo. } \\
\text { 5. Prioriza los estudios de me- } \\
\text { jora de la política de man- } \\
\text { tenimiento a los modos de } \\
\text { fallo más críticos. }\end{array}$ & $\begin{array}{l}1 \rightarrow 2 \text { Concepto de criticidad en } \\
\text { mantenimiento. } \\
2 \rightarrow 3 \text { Componentes de la } \\
\text { criticidad. } \\
3 \rightarrow 4 \text { Relación de la criticidad con } \\
\text { distintos aspectos del riesgo. } \\
4 \rightarrow 5 \text { Se prioriza la actuación } \\
\text { para los modos de fallo de más } \\
\text { criticidad. }\end{array}$ \\
\hline 5 & $\begin{array}{l}\text { 1. No responde nada o la con- } \\
\text { 2. Destación no concuerda. } \\
\text { de un equipo. } \\
\text { 3. Técnicas para medir la con- } \\
\text { dición de un equipo. } \\
\text { 4. Definición y establecimiento } \\
\text { de los límites de alarma de } \\
\text { la condición del equipo. } \\
\text { 5. Elección del tipo de tarea de } \\
\text { mantenimiento en función } \\
\text { a la condición del equipo } \\
\text { monitorizada. }\end{array}$ & $\begin{array}{l}1 \rightarrow 2 \text { Definir condición de un } \\
\text { equipo. } \\
2 \rightarrow 3 \text { Técnicas para detectar la } \\
\text { condición de un equipo. } \\
3 \rightarrow 4 \text { Saber establecer alarmas } \\
\text { para la señal monitorizada. } \\
4 \rightarrow 5 \text { Elegir la tarea de manteni- } \\
\text { miento acorde a la condición de } \\
\text { equipo. }\end{array}$ \\
\hline 6 & $\begin{array}{l}\text { 1. No responde nada o la con- } \\
\text { testación concuerda. } \\
\text { 2. Conceptos de técnicas de } \\
\text { análisis de la condición y de } \\
\text { análisis de fallos. } \\
\text { 3. Tipo de información que } \\
\text { aportan ambas técnicas en } \\
\text { relación a los datos de fallo. } \\
\text { 4. Ubicación temporal de am- } \\
\text { bas técnicas. } \\
\text { 5. Para qué se usan los resul- } \\
\text { tados de ambas técnicas. }\end{array}$ & $\begin{array}{l}1 \rightarrow 2 \text { Definición de condición y } \\
\text { fallo. } \\
2 \rightarrow 3 \text { La condición es un dato, de } \\
\text { los fallos obtenemos datos. } \\
3 \rightarrow 4 \text { La condición es previa al fa- } \\
\text { llo, los datos de fallo posteriores. } \\
4 \rightarrow 5 \text { Para determinar el punto } \\
\text { óptimo de mantenimiento. }\end{array}$ \\
\hline
\end{tabular}

Jornadas de Formación e Innovación Docente del Profesorado | № 2 (2019) Esta obra se distribuye con la licencia Creative Commons 


\begin{tabular}{|c|c|c|c|}
\hline 7 & $\begin{array}{l}1 . \\
2 . \\
3 . \\
4 . \\
5 .\end{array}$ & $\begin{array}{l}\text { No responde nada o la con- } \\
\text { testación no concuerda. } \\
\text { Definición escasa de téc- } \\
\text { nicas de análisis de la } \\
\text { condición. } \\
\text { Definición completa de } \\
\text { técnicas de análisis de la } \\
\text { condición. } \\
\text { Algunos casos en los que } \\
\text { están indicadas. } \\
\text { Se indican los casos en los } \\
\text { que están indicadas. }\end{array}$ & $\begin{array}{l}\text { 1 } \rightarrow 2 \text { Conoce la definición de téc- } \\
\text { nicas de análisis de la condición. } \\
2 \rightarrow 3 \text { Dominio de la definición. } \\
3 \rightarrow 4 \text { Sabe aplicar algunas técni- } \\
\text { cas análisis de la condición. } \\
4 \rightarrow 5 \text { Domina la aplicación } \\
\text { de técnicas de análisis de la } \\
\text { condición. }\end{array}$ \\
\hline 8 & & $\begin{array}{l}\text { No responde nada o la con- } \\
\text { testación no concuerda. } \\
\text { Definen algunas tareas de } \\
\text { mantenimiento. } \\
\text { Definen los tipos de tareas } \\
\text { de mantenimiento. } \\
\text { Establece algunos criterios } \\
\text { de decisión de la tarea más } \\
\text { conveniente. } \\
\text { Establece los criterios de } \\
\text { decisión para cada tarea de } \\
\text { mantenimiento. }\end{array}$ & $\begin{array}{l}1 \rightarrow 2 \text { Conoce algunos de los tipos } \\
\text { de tareas de mantenimiento. } \\
2 \rightarrow 3 \text { Conoce todos los tipos de } \\
\text { tareas. } \\
3 \rightarrow 4 \text { Conoce algunos criterios de } \\
\text { decisión. } \\
4 \rightarrow 5 \text { Conoce los criterios de de- } \\
\text { cisión según el tipo de tarea. }\end{array}$ \\
\hline
\end{tabular}

Uno de los objetivos del profesor durante el desarrollo de las actividades fue la de trabajar indirectamente las preguntas del cuestionario.

Tras la evaluación se observa una clara mejoría en el nivel de aprendizaje. En las Figuras 2 a 9 se representa la comparación de los niveles de aprendizaje de la clase en cada una de las ocho cuestiones de los cuestionarios inicial y final. En la Figura 10 se recoge una valoración media para cada cuestión y en general para el conjunto de los cuestionarios. Los números presentes en las figuras representan el número de alumnos que responde en cada nivel. 
Cuestión 1

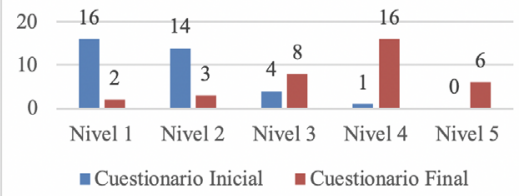

Figura 2. $\mathrm{N}^{\circ}$ de alumnos por nivel. Cuestión 1. Cuestión 3

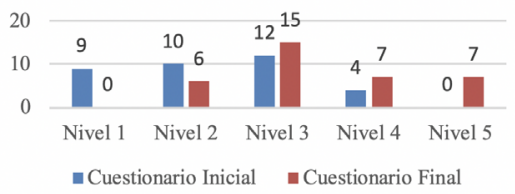

Figura 4. $N^{\circ}$ de alumnos por nivel. Cuestión 3.

\section{Cuestión 2}

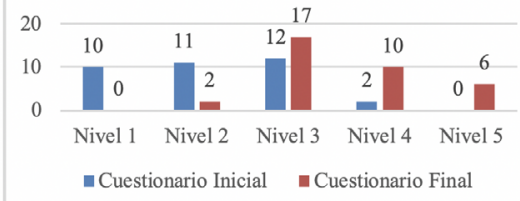

Figura 3. $\mathrm{N}^{\circ}$ de alumnos por nivel. Cuestión 2.

Cuestión 4

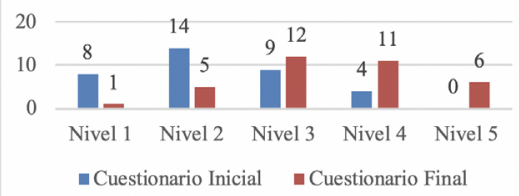

Figura 5. $\mathrm{N}^{\circ}$ de alumnos por nivel. Cuestión 4.

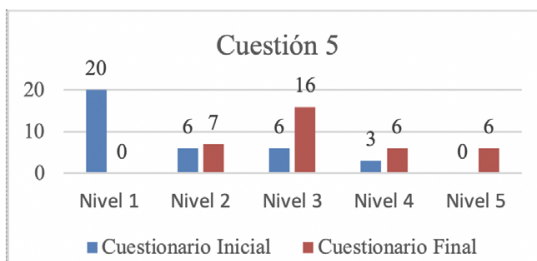

Figura 6. $\mathrm{N}^{\circ}$ de alumnos por nivel. Cuestión 5.

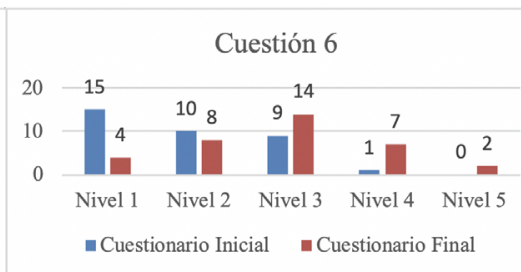

Figura 7. $\mathrm{N}^{\mathrm{0}}$ de alumnos por nivel. Cuestión 6.

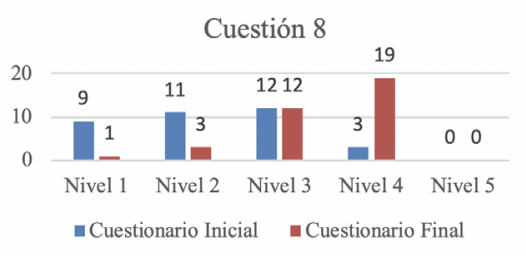

Figura 9. $\mathrm{N}^{\circ}$ de alumnos por nivel. Cuestión 8.

Figura $8 . \mathrm{N}^{\circ}$ de alumnos por nivel. Cuestión 7.

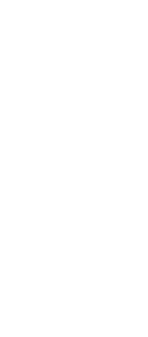

\section{Cuestión 7}
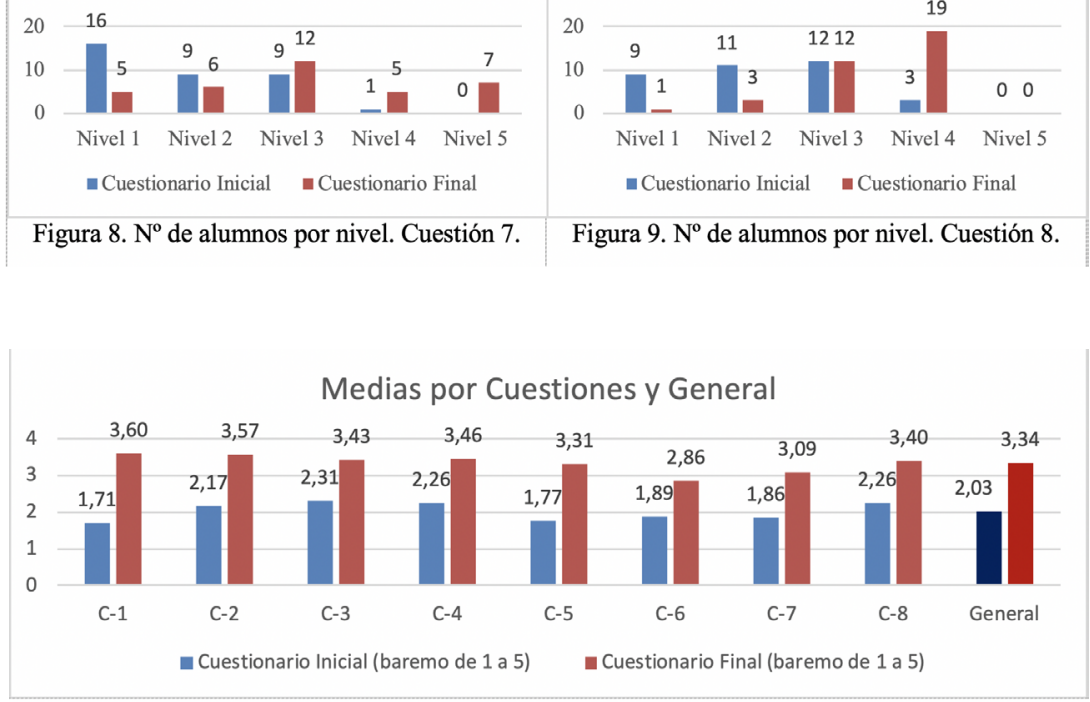

Figura 10. Valoración media en cada cuestión y media general. 


\section{ANTONIO SÁNCHEZ HERGUEDAS}

\section{Valoración individual del aprendizaje}

Para analizar el aprendizaje individualizado para cada alumno, se ha elaborado la Tabla 5. En ella se reflejan las valoraciones (escalón en el que se sitúan) en cada cuestión de los cuestionarios inicial y final de los treinta y cinco alumnos que han realizado las ocho actividades y los dos cuestionarios. A los otros siete alumnos no representados les ha faltado alguna de estas pruebas.

Tabla 5. Evaluación individualizada por alumno para cada una de las cuestiones.

\begin{tabular}{|c|c|c|c|c|c|c|c|c|c|c|c|c|c|c|c|c|c|c|c|}
\hline \multirow[b]{2}{*}{ Alumno } & \multicolumn{9}{|c|}{ Cuestionario Inicial (baremo de 1 a 5) } & \multicolumn{10}{|c|}{ Cuestionario Final (baremo de 1 a 5) } \\
\hline & $C-1$ & $C-2$ & $C-3$ & $C-4$ & $C-5$ & $C-6$ & $C-7$ & $C-8$ & Media & $C-1$ & $C-2$ & $C-3$ & $C-4$ & $C-5$ & $C-6$ & $\mathrm{C}-7$ & $C-8$ & Media & Dif. \\
\hline 1 & 2 & 1 & 3 & 2 & 4 & 2 & 4 & 3 & 2,63 & 4 & 3 & 3 & 2 & 3 & 3 & 4 & 3 & 3,13 & 0,5 \\
\hline 2 & 1 & 3 & 1 & 1 & 1 & 1 & 1 & 1 & 1,25 & 2 & 5 & 3 & 3 & 2 & 1 & 2 & 2 & 2,5 & 1,25 \\
\hline 3 & 1 & 3 & 2 & 2 & 3 & 2 & 3 & 2 & 2,25 & 4 & 3 & 5 & 4 & 3 & 5 & 3 & 4 & 3,88 & 1,63 \\
\hline 4 & 2 & 1 & 3 & 4 & 3 & 4 & 3 & 2 & 2,75 & 5 & 3 & 2 & 4 & 3 & 4 & 5 & 3 & 3,63 & 0,88 \\
\hline 5 & 1 & 2 & 3 & 2 & 3 & 3 & 1 & 3 & 2,25 & 4 & 5 & 3 & 2 & 3 & 3 & 3 & 4 & 3,38 & 1,13 \\
\hline 6 & 1 & 1 & 2 & 1 & 1 & 2 & 3 & 2 & 1,63 & 3 & 3 & 5 & 4 & 5 & 3 & 3 & 4 & 3,75 & 2,13 \\
\hline 7 & 2 & 2 & 3 & 4 & 1 & 3 & 2 & 2 & 2,38 & 4 & 3 & 3 & 4 & 3 & 4 & 4 & 3 & 3,5 & 1,13 \\
\hline 8 & 1 & 2 & 2 & 1 & 1 & 1 & 1 & 1 & 1,25 & 4 & 5 & 3 & 5 & 3 & 2 & 2 & 3 & 3,38 & 2,13 \\
\hline 9 & 3 & 1 & 3 & 2 & 1 & 2 & 2 & 3 & 2,13 & 3 & 5 & 4 & 4 & 3 & 3 & 3 & 3 & 3,5 & 1,38 \\
\hline 10 & 1 & 2 & 2 & 2 & 1 & 2 & 1 & 1 & 1,5 & 4 & 3 & 4 & 3 & 5 & 3 & 3 & 3 & 3,5 & 2 \\
\hline 11 & 2 & 3 & 3 & 2 & 3 & 3 & 2 & 3 & 2,63 & 5 & 4 & 3 & 5 & 4 & 3 & 5 & 4 & 4,13 & 1,5 \\
\hline
\end{tabular}




\section{ANTONIO SÁNCHEZ HERGUEDAS}

\begin{tabular}{|c|c|c|c|c|c|c|c|c|c|c|c|c|c|c|c|c|c|c|c|}
\hline 12 & 2 & 3 & 3 & 3 & 2 & 3 & 2 & 2 & 2,5 & 3 & 3 & 4 & 3 & 3 & 2 & 5 & 4 & 3,38 & 0,88 \\
\hline 13 & 3 & 3 & 2 & 2 & 2 & 2 & 1 & 4 & 2,38 & 4 & 5 & 3 & 4 & 4 & 2 & 3 & 4 & 3,63 & 1,25 \\
\hline 14 & 4 & 4 & 3 & 3 & 4 & 3 & 3 & 3 & 3,38 & 5 & 4 & 2 & 3 & 5 & 2 & 4 & 4 & 3,63 & 0,25 \\
\hline 15 & 2 & 4 & 3 & 2 & 4 & 1 & 2 & 3 & 2,63 & 5 & 3 & 3 & 5 & 4 & 4 & 4 & 4 & 4 & 1,38 \\
\hline 16 & 1 & 3 & 2 & 2 & 1 & 1 & 2 & 3 & 1,88 & 4 & 5 & 3 & 5 & 2 & 2 & 5 & 3 & 3,63 & 1,75 \\
\hline 17 & 2 & 3 & 4 & 3 & 3 & 3 & 2 & 3 & 2,88 & 5 & 3 & 3 & 3 & 3 & 4 & 5 & 4 & 3,75 & 0,88 \\
\hline 18 & 2 & 2 & 1 & 3 & 1 & 2 & 1 & 1 & 1,63 & 3 & 3 & 3 & 1 & 2 & 3 & 1 & 1 & 2,13 & 0,5 \\
\hline 19 & 1 & 3 & 1 & 4 & 2 & 1 & 1 & 1 & 1,75 & 2 & 3 & 2 & 4 & 3 & 1 & 1 & 3 & 2,38 & 0,63 \\
\hline 20 & 1 & 3 & 1 & 2 & 1 & 1 & 1 & 2 & 1,5 & 3 & 3 & 4 & 4 & 2 & 3 & 2 & 4 & 3,13 & 1,63 \\
\hline 21 & 2 & 1 & 1 & 3 & 1 & 1 & 1 & 2 & 1,5 & 4 & 4 & 5 & 2 & 2 & 2 & 1 & 2 & 2,75 & 1,25 \\
\hline 22 & 1 & 2 & 2 & 2 & 3 & 3 & 3 & 3 & 2,38 & 4 & 4 & 5 & 5 & 5 & 3 & 3 & 4 & 4,13 & 1,75 \\
\hline 23 & 3 & 1 & 4 & 3 & 2 & 2 & 3 & 4 & 2,75 & 4 & 4 & 4 & 3 & 4 & 4 & 4 & 4 & 3,88 & 1,13 \\
\hline 24 & 3 & 3 & 1 & 1 & 1 & 1 & 3 & 1 & 1,75 & 3 & 3 & 2 & 4 & 4 & 1 & 3 & 3 & 2,88 & 1,13 \\
\hline 25 & 1 & 1 & 4 & 1 & 1 & 1 & 1 & 1 & 1,38 & 2 & 3 & 3 & 4 & 3 & 4 & 2 & 3 & 3 & 1,63 \\
\hline 26 & 1 & 2 & 2 & 1 & 2 & 2 & 2 & 4 & 2 & 5 & 2 & 3 & 5 & 2 & 5 & 3 & 3 & 3,5 & 1,5 \\
\hline 27 & 2 & 2 & 3 & 4 & 1 & 1 & 1 & 1 & 1,88 & 1 & 2 & 2 & 3 & 3 & 4 & 1 & 2 & 2,25 & 0,38 \\
\hline 28 & 2 & 2 & 1 & 3 & 1 & 1 & 1 & 2 & 1,63 & 4 & 3 & 2 & 3 & 5 & 2 & 3 & 4 & 3,25 & 1,63 \\
\hline 29 & 2 & 3 & 1 & 3 & 1 & 2 & 1 & 2 & 1,88 & 4 & 4 & 5 & 4 & 3 & 3 & 5 & 4 & 4 & 2,13 \\
\hline 30 & 2 & 1 & 1 & 1 & 1 & 1 & 1 & 2 & 1,25 & 3 & 4 & 5 & 3 & 5 & 1 & 1 & 3 & 3,13 & 1,88 \\
\hline 31 & 1 & 2 & 3 & 2 & 1 & 1 & 1 & 3 & 1,75 & 4 & 4 & 4 & 3 & 3 & 2 & 3 & 4 & 3,38 & 1,63 \\
\hline 32 & 1 & 2 & 2 & 2 & 1 & 1 & 3 & 2 & 1,75 & 3 & 4 & 4 & 2 & 2 & 3 & 2 & 4 & 3 & 1,25 \\
\hline 33 & 1 & 3 & 2 & 1 & 1 & 3 & 1 & 1 & 1,63 & 4 & 3 & 3 & 3 & 4 & 3 & 2 & 4 & 3,25 & 1,63 \\
\hline & & & & & & & & & & & & \\
\hline
\end{tabular}

Jornadas de Formación e Innovación Docente del Profesorado | № 2 (2019)

(C) Esta obra se distribuye con la licencia Creative Commons

Reconocimiento-NoComercial-SinObraDerivada

Internacional (CC BY-NC-ND 4.0.) 


\section{ANTONIO SÁNCHEZ HERGUEDAS}

\begin{tabular}{|l|l|l|l|l|l|l|l|l|l|l|l|l|l|l|l|l|l|l|l|l|}
\hline 34 & 2 & 1 & 4 & 3 & 1 & 1 & 3 & 3 & 2,25 & 4 & 4 & 5 & 2 & 3 & 3 & 5 & 4 & 3,75 & 1,5 \\
\hline 35 & 1 & 1 & 3 & 2 & 2 & 3 & 2 & 3 & 2,13 & 1 & 3 & 3 & 3 & 3 & 3 & 3 & 4 & 2,88 & 0,75 \\
\hline & & & & & \multicolumn{6}{|c|}{ Media Global } & 2,03 & & & & & & Media Global & 3,34 & 1,31 \\
\hline
\end{tabular}

La diferencia de conocimiento inicial y final se representa en las gráficas de las Figuras 11 y 12. Se observa que hay un aumento medio de 1,3 obstáculos. Existen 16 respuestas que retroceden frente a 199 que avanzan, posiblemente debido a fallos de atención durante la realización del cuestionario (preguntados esos alumnos, su contestación no reflejó el nivel expresado, sino otro superior).

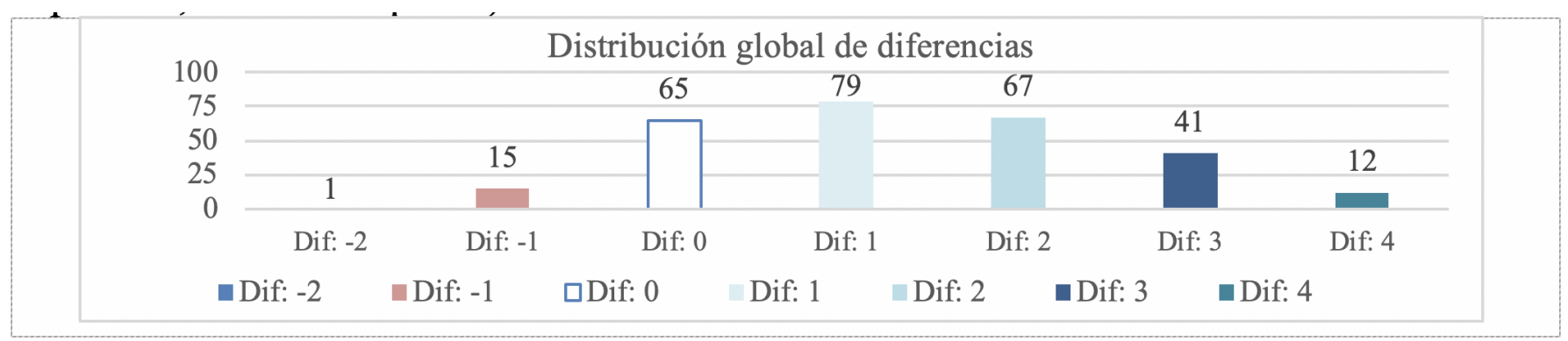

Figura 11. Representación de las diferencias de nivel de conocimiento global. 
ANTONIO SÁNCHEZ HERGUEDAS

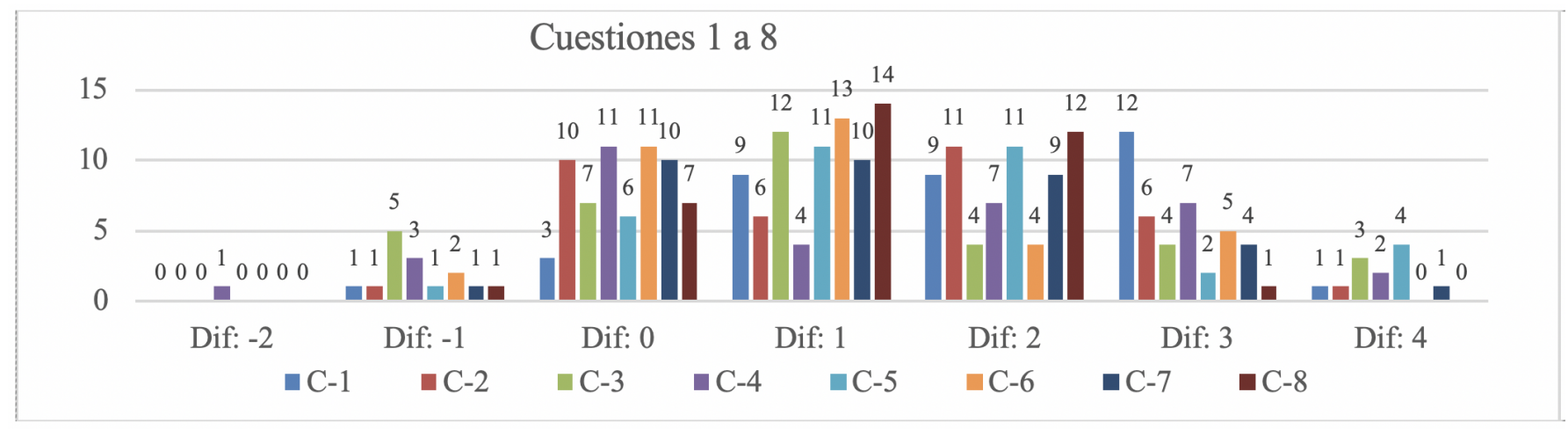

Figura 12. Representación de las diferencias de nivel de conocimiento por cuestiones. 


\section{Evaluación del Ciclo de Mejora en el Aula}

Evaluando la experiencia en el CIMA, he descubierto la ayuda que supone desarrollar mapas de contenidos, no solo por la recopilación de conceptos, procedimientos y actitudes sino porque aportan seguridad en el proceso del ciclo. También, en la medida de lo posible, es bueno utilizar actividades donde se resuelvan problemas cotidianos que sean extrapolables al problema de la disciplina, buscando la ayuda para la comprensión y para futuras actualizaciones del profesional que se está formando. Para valorar el progreso de aprendizaje de los alumnos son imprescindibles los cuestionarios. Su diseño debe localizar los obstáculos al aprendizaje, de manera que seamos capaces de establecer distintos niveles de conocimiento, por la superación ordenada de estos obstáculos. En esa línea va la aproximación que he intentado hacer anteriormente.

Durante las explicaciones teóricas de la asignatura, sólo se pondrá atención en aquellos aspectos más relevantes o dificiles de entender, dejando otros aspectos para el estudio individual de los alumnos. Este ahorro de tiempo se utilizará en las actividades.

Por último, es necesario establecer relaciones de confianza con los alumnos, de manera que éstos dejen aflorar sus ideas e inquietudes y al mismo tiempo vean en el profesor aquel maestro que les enseña, dirige y anima.

Jornadas de Formación e Innovación Docente del Profesorado | № 2 (2019) Esta obra se distribuye con la licencia Creative Commons Reconocimiento-NoComercial-SinObraDerivada 


\section{Referencias bibliográficas}

Bain, K. (2007). Lo que hacen los mejores profesores de universidad. Valencia: Publicacions de la Universitat de València.

De Alba, N. y Porlán, R. (2017). La metodología de enseñanza (pp. 23-36). En R. Porlán (Coord.), Enseñanza Universitaria. Cómo mejorarla. Madrid: Morata.

Delval, J. A. (2001). Aprender en la vida y en la escuela. Madrid: Morata.

Esteve Zarazaga, J. (2007). Un examen a la cultura escolar. Avances en Supervisión Educativa. Revista de la Asociación de Inspectores de Educación de España, 7. Recuperado de https://avances.adide.org/index.php/ase/ article/view 295

Finkel, D. L. (2008). Dar clase con la boca cerrada. Publicacions de la Universitat de València.

Rivero, A. y Porlán, R. (2017). La evaluación en la enseñanza universitaria (pp.73-91). En R. Porlán (Ed.), Enseñanza universitaria. Como mejorarla. Madrid: Morata.

Jornadas de Formación e Innovación Docente del Profesorado | № 2 (2019) Esta obra se distribuye con la licencia Creative Commons 